\section{ORIGINAL} RESEARCH

\author{
D.F. Black \\ A.E. Rad \\ L.A. Gray \\ N.G. Campeau \\ D.F. Kallmes
}

\title{
Cerebral Venous Sinus Density on Noncontrast CT Correlates with Hematocrit
}

BACKGROUND AND PURPOSE: A positive correlation between HCT and CT attenuation of intravascular blood has long been assumed but has never been established by using substantial patient numbers and modern CT equipment. The purpose of this study was to determine whether apparent increased attenuation on $\mathrm{CT}$ in cerebral venous sinuses can be attributed to hemoconcentration alone and to assess whether sinus thrombosis can be differentiated from hemoconcentrated blood based on attenuation values alone.

MATERIALS AND METHODS: We measured HUs in a region of interest within the confluence of dural venous sinuses in 166 unenhanced head CTs and correlated these data with HCT and HGB values in male and female patients aged 2 to 100 years. We then compared these data with similar measurements in 8 patients with recent venous sinus thrombosis. Two-tailed $t$ test and linear regression analyses were performed to evaluate HGB and HCT between groups and with measured CT attenuation of intravascular blood, respectively.

RESULTS: A statistically significant relationship was noted between both HCT and HGB with CT attenuation. Seven of 8 patients with sinus thrombosis had attenuation values $>70$, but none of the normal subjects had HUs $>70$.

CoNCLUSIONS: Hemoconcentration correlates with CT attenuation in cerebral venous sinuses. Our findings suggest that comparing the ratio of HUs to HCT may be useful in gauging concern for sinus thrombosis.

ABBREVIATIONS: $\mathrm{HCT}=$ hematocrit; $\mathrm{HGB}=$ hemoglobin; $\mathrm{H}: \mathrm{H}=$ Hounsfield unit-to-hematocrit ratio; $\mathrm{HU}=$ Hounsfield unit; $\mathrm{MRV}=\mathrm{MR}$ venography; $\mathrm{ROI}=$ region of interest; $\mathrm{SSS}=$ superior sagittal sinus

I n our routine practice, we have observed that radiodensity or attenuation on CT images within the intracranial vasculature may vary greatly among patients. In some cases, elevated radiodensity within a given vascular structure may lead to the suspicion of vascular thrombosis. The concept of "hemoconcentration" or elevated levels of HGB or HCT leading to increased $x$-ray attenuation within the vasculature is widely accepted. ${ }^{1}$ However, the data substantiating such a correlation are fairly weak, being limited to a small in vitro study and 3 clinical studies comprising 67 patients in total. ${ }^{2-5}$

To better characterize the correlation between $\mathrm{x}$-ray attenuation and HGB/HCT levels on CT, we carried out a prospective study in a large, consecutive series of patients and compared these data to findings in a cohort of patients with documented dural sinus thrombosis. Improved understanding of the correlation between hemoconcentration and CT attenuation within the dural sinuses may improve triage of patients to or from further work-up of suspected sinus thrombosis.

The purpose of this study was to determine whether apparent increased attenuation on CT within cerebral venous sinuses can be attributed to hemoconcentration.

Received October 24, 2010; accepted after revision December 8 .

From the Department of Radiology, Mayo Clinic, Rochester, Minnesota.

Please address correspondence to David F. Black, Mayo Clinic, W2, 200 First St SW, Rochester MN 55905; e-mail: black.david@mayo.edu

DOI 10.3174/ajnr.A2504

\section{Materials and Methods}

This study was approved by our institutional review board before beginning data collection. Between month 1 and month 2 in 2008, we analyzed consecutive, unenhanced head CTs in our inpatient/emergency department practice. Patients were excluded if they had any intracranial artifact, pathology, trauma (eg, intracranial hemorrhage or calvarial fracture), or operative lesions that might obscure or affect the venous sinus system. Patients were not required to have completely negative studies-only findings that the authors thought could obscure or alter $\mathrm{x}$-ray attenuation measurements at the venous confluence or inferior aspect of the superior sagittal sinus resulted in exclusion. Patients also were excluded if they had received contrast material for another study within 3 days of the CT or if they were suspected, based on clinical findings, of having a venous sinus thrombosis. Only those patients with a complete blood count drawn within 24 hours of the CT were included. Patients were imaged on 1 of 264section CT scanners (Somatom Sensation 64; Siemens Medical Solutions, Forchheim, Germany). Images were acquired by using CT parameters of $120 \mathrm{kV}$; section thickness, $5 \mathrm{~mm} ; 35-570 \mathrm{~mA}$; and pitch, 1 . The final data base included 166 subjects ( 83 men, 83 women).

To ensure reproducibility in our methods, the first 26 consecutive subjects were reviewed independently by 1 of 3 experienced observers (D.F.B., A.E.R., D.F.K.) by using Advantage Workstation version 4.3 (GE Healthcare, Milwaukee, Wisconsin). An ROI was drawn by using a cursor within the inferior aspect of the superior sagittal sinus in most cases, but in some, the ROI was drawn in the confluence of the venous sinuses if this happened to yield a larger region of clearly visualized sinus. In addition, $5 \mathrm{ROI}$ circles of varying volume, ranging from 5 to $9 \mathrm{~mm}^{2}$, were taken in each of the first 26 subjects to assess for any significant variability. HU values were very similar even with different-sized ROIs. But because sinus size varies greatly from pa- 

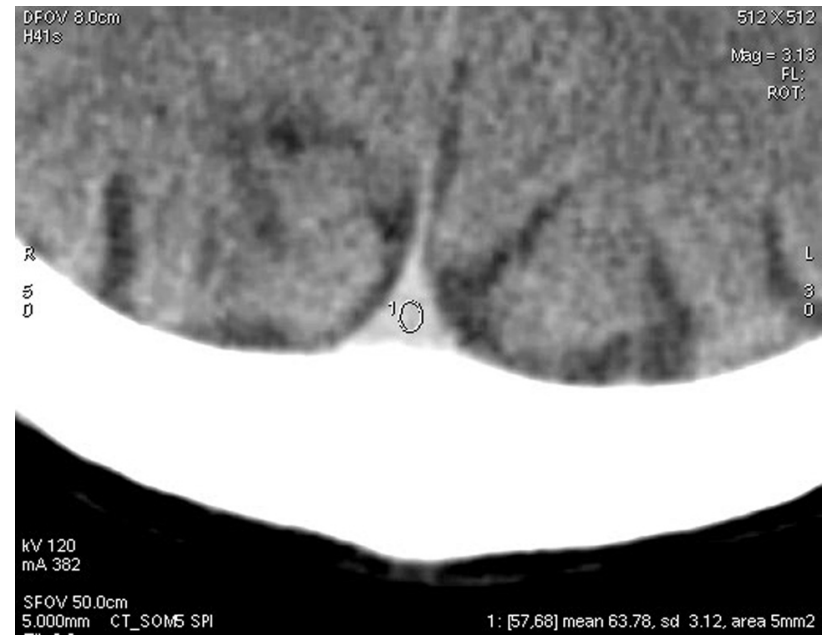

Fig 1. An example to demonstrate region of interest placement within the inferior aspect of the superior sagittal sinus.

tient to patient, and a larger ROI, as long as it does not include the sinus wall or artifact from the adjacent calvaria, is more likely to be an accurate representation of reality than a tiny ROI, we chose to use a "best fit" model wherein we manually created a circular or elliptical ROI making it as large as possible without overlapping with the sinus wall (Fig 1). We felt this would be a more realistic model in clinical practice as well. Subsequent to this, reviewer 1 used this technique to measure the remaining subjects.

In addition, we compared 8 cases of documented sinus thrombosis with our subjects to determine intrasinus attenuation. All of these patients underwent MRV to confirm initial suspicions based both on high attenuation in the venous sinuses on noncontrast head CTs and clinical presentation. One subject with proved sinus thrombosis was excluded because a well-defined venous sinus to measure distinct from calvaria could not be identified on the images obtained. HU measurements for subjects with known sinus thromboses were made by using identical methods, except that the ROIs were centered over the specific segments shown to be thrombosed on MR angiography, conventional venography, or both.

To normalize observed blood attenuation with regard to hemoconcentration, we calculated the $\mathrm{H}: \mathrm{H}$ ratio for each patient.

\section{Statistical Analysis}

A 2-tailed $t$ test was performed to compare age, HGB, and HCT between patients with and without thrombosis. A linear regression analysis was used to asses the correlation between HGB and HCT with measured CT attenuation of intravascular blood. We also compared

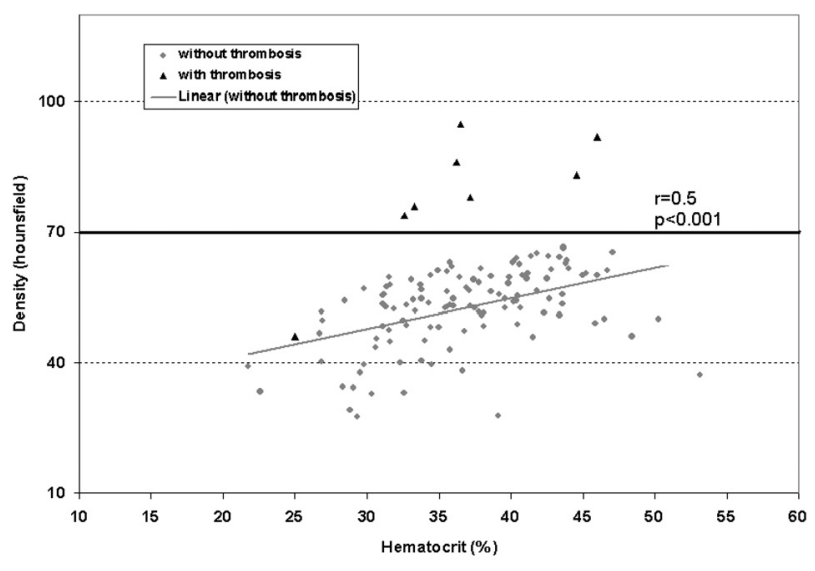

Fig 2. Scatterplot of sinus attenuation as a function of HCT with an HU $>70$ discriminating almost all cases with thrombosis from those without.

the $\mathrm{H}: \mathrm{H}$ ratios between patients with and without sinus thrombosis by using a 2 -tailed $t$ test.

\section{Results}

Overall ages in the 166 subjects ranged from 2 to 100 years, with an average age of 56.9 years, and a SD of 23.6 years. Female ages ranged from 2 to 100 years, with an average age of 59 (SD 24.7) years. Male ages ranged from 4 to 95 years, with an average age of 55 (SD 22.3) years.

Mean ROI areas and SDs in the first 26 subjects were 5.0 $\mathrm{mm}^{2}(1.3), 5.3 \mathrm{~mm}^{2}(1.6)$, and $4.7 \mathrm{~mm}^{2}(0.9)$ for reviewers 1,2 , and 3, respectively. Measuring HUs with different-sized ROIs in the sinus altered the values by only 3 HUs on average (SD 3.2).

For the 166 patients imaged prospectively, the mean (SD) HUs ranged from 32 to 69 (1.79-9.12). For men, HUs ranged from 36 to 69 (1.79-9.12), and the values for women ranged from 32 to 66 (1.81-7.37). HCT values (SD) ranged from $23 \%$ to $45.6 \%$ (16.3) in women and from $24 \%$ to $53 \%$ (19.4) in men. HBG values ranged from 8.1 to $15.9(5.9)$ in women and from 8.1 to 18.4 (6.9) in men.

A statistically significant correlation between HGB and HCT values with HUs was found for both men and women. There was no statistically significant difference in maximum, minimum, or mean values for age, HCT, or HGB between men and women. For all correlations, the $P$ value was $<.0001$.

The 8 included cases of documented sinus thrombosis had HU values ranging from 46 to 95, with a mean of 77 (Table). Figure 2 depicts the CT attenuation of venous sinuses as a function of HCT and shows that an HU $>70$ separates all cases

\begin{tabular}{|c|c|c|c|c|c|c|c|}
\hline Attenua & In and hemocc & entration parameters for 8 patients & th MRV-confirmed acut & enous sinus th & nboses & & \\
\hline Subject & Age/Gender & Thrombus Site & $\begin{array}{l}\text { Thrombus Age (days) } \\
\text { at Time of CT }\end{array}$ & $\begin{array}{c}\text { HU } \\
(\text { Mean }=77)\end{array}$ & НСТ\% & $\begin{array}{c}\text { H:H Ratio } \\
(\text { Mean }=2.20)\end{array}$ & HGB \\
\hline 1 & $29 / \mathrm{M}$ & SSS & 3 & 92 & 46 & 2.0 & 15.9 \\
\hline 2 & $26 / F$ & SSS & 4 & 86 & 36.2 & 2.38 & 11.8 \\
\hline 3 & $39 / M$ & SSS, torcula & 7 & 83 & 44.6 & 1.86 & 15.4 \\
\hline 4 & $53 / \mathrm{F}$ & $\begin{array}{l}\text { Straight, bilateral transverse, sigmoid, } \\
\text { torcula, SSS }\end{array}$ & 3 & 78 & 37.2 & 2.10 & 12.8 \\
\hline 5 & $41 / F$ & Left transverse, sigmoid & 3 & 74 & 32.6 & 2.27 & 11.3 \\
\hline 6 & $41 / M$ & SSS, left transverse & 1 & 76 & 33.3 & 2.28 & 11.1 \\
\hline 7 & $50 / F$ & SSS, straight, left transverse & 2 & 95 & 36.5 & 2.60 & 12.7 \\
\hline 8 & $18 / \mathrm{M}$ & SSS, straight, bilateral transverse & 12 & 46 & 25.0 & 1.84 & 8.6 \\
\hline
\end{tabular}

Note:-Age is defined as the estimated time interval between onset of symptoms from thrombosis and measuring attenuation of thrombosis on CT. 


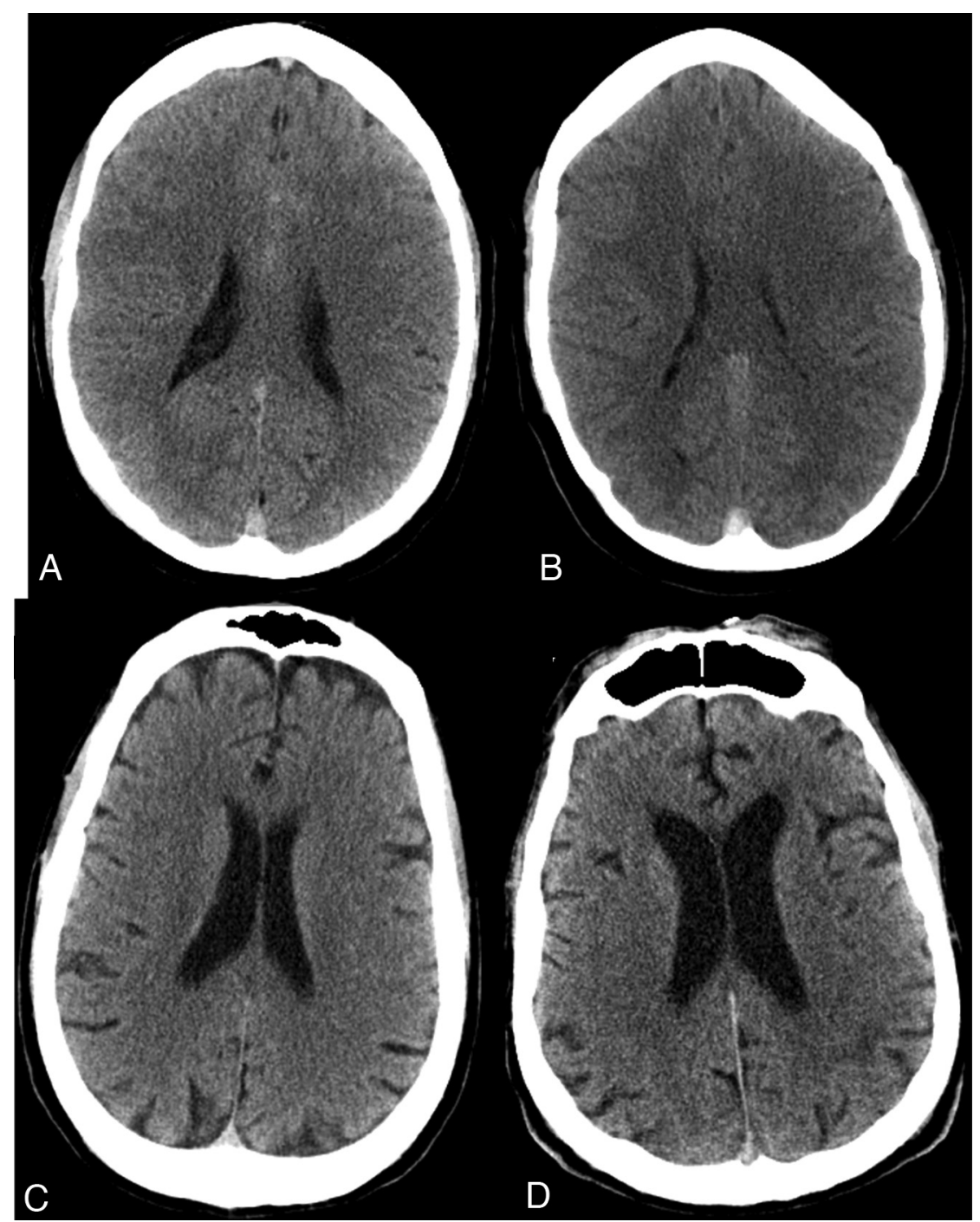

Fig 3. Unenhanced CT of subjects $A$ (HU 92, HCT46) and $B$ (HU 86, HCT 36), both with documented superior sagittal sinus thromboses, compared with subjects $C$ (HU 66, HCT 43) and $D$ (HU 64, HCT 43), without thrombosis. Note that hemoconcentration may mimic the appearance of a sinus thrombosis.

with thrombosis from those without thrombosis except 1. Figure 3 demonstrates that on visual inspection, it can be difficult to distinguish between subjects with and without sinus thrombosis on noncontrast CT scans.

In the 166 subjects without thromboses, the $\mathrm{H}: \mathrm{H}$ ratio ranged from 0.69 to $1.91(0.69-1.91$ in women and $0.90-1.90$ in men), with a mean of 1.44 . In the 8 subjects with venous sinus thrombosis, the $\mathrm{H}: \mathrm{H}$ ratio ranged from 1.84 to 2.60 , with a mean of 2.20 (Fig 4).

\section{Discussion}

This report confirms the long-held belief that intravascular radiodensity correlates with degree of hemoconcentration. We demonstrated that $\mathrm{x}$-ray attenuation is highly correlated with both HGB and HCT levels. This is important because the hemoconcentration may cause CT attenuation to appear as if patients have thrombosis when they in fact do not. Furthermore, in 7 of 8 cases with sinus thrombosis, the $\mathrm{HU}$ was $>70$. The highest HU among our cohort without sinus thrombosis was 69; thus, if the HU over an attenuated region of venous sinus is $>70$, further investigations to exclude a venous sinus

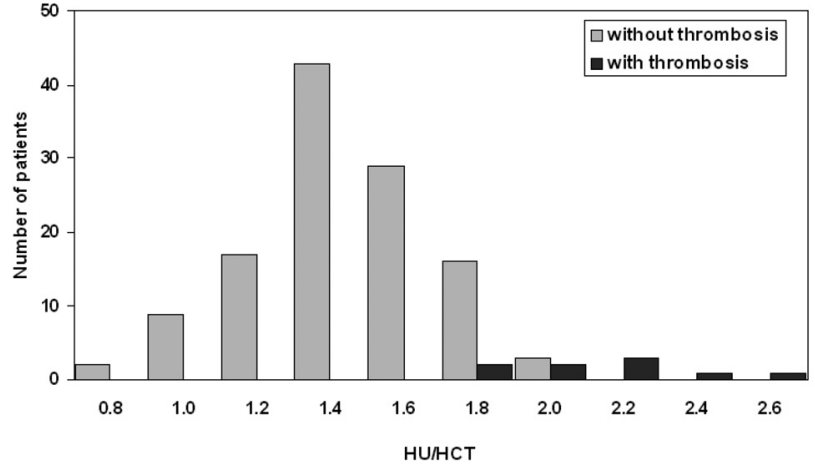

Fig 4. H:H ratio distribution demonstrates very little overlap between values for subjects without and with thrombosis, with no overlap when the value is $\geq 2.0$.

thrombosis may be warranted. This degree of attenuation has been found in acute clots elsewhere in the body. ${ }^{6}$ The lowest $\mathrm{HU}$ value in our cohort of patients with sinus thrombosis was 46 , but this may be low because it was a much "older" clot at the time the $\mathrm{HU}$ was measured (12 days after symptom onset). 
This thrombosis also was shown to be a nonocclusive clot on conventional angiography; thus, volume averaging may have reduced the HU value.

Finally, by using a normalized ratio of HU to HCT, we have shown in our cohort that the greater the difference between a high HU value and a low HCT value, the more likely a patient is to have a sinus thrombosis and further confirmatory imaging studies should be recommended. By quantifying the discrepancy between $\mathrm{x}$-ray attenuation and $\mathrm{HCT}$, the $\mathrm{H}: \mathrm{H}$ ratio provides another method to gauge concern for sinus thrombosis beyond the maximum $\mathrm{HU}$ measured. The mean $\mathrm{H}: \mathrm{H}$ value in cases with thrombosis was 2.20 versus 1.44 in subjects without clot. The distribution of $\mathrm{H}: \mathrm{H}$ ratios between subjects with and without sinus thromboses demonstrates very little overlap. In our small cohort, a conservative threshold of an $\mathrm{H}: \mathrm{H}$ ratio of $\geq 2.0$ suggests a strong likelihood of clot, but even an $\mathrm{H}: \mathrm{H}$ ratio of 1.8-1.9 may warrant further evaluation in the proper clinical context. Only 7 (4\%) of 166 subjects without thrombosis had $\mathrm{H}: \mathrm{H}$ ratios of $1.8-1.9$ and none were $\geq 2.0$.

Correlation between $\mathrm{x}$-ray attenuation and hemoconcentration has been noted previously in 1 study wherein HGB and HU were found to correlate in 33 patients by using unenhanced CTs of the chest with an ROI in the aorta in an attempt to diagnose anemia based on $\mathrm{x}$-ray attenuation. ${ }^{2}$

New and Aronow ${ }^{4}$ used blood product preparations to demonstrate an in vitro correlation between HU and HCT, whereas the retrospective study of 10 noncontrast head CTs by Ben Salem et $\mathrm{al}^{3}$ concluded that an HCT $>60 \%$ produced attenuated-appearing vasculature. Nelson et $\mathrm{al}^{5}$ reported 15 patients with attenuated-appearing dural sinuses on CT without thrombosis, and many of these had elevated HGBs or HCTs. Other case reports indicate that patients with high HCTs, usually those with polycythemia vera, may have very high attenuation within the dural sinuses on unenhanced CT, mimicking the appearance of sinus thrombosis. ${ }^{7}$ Still other case reports demonstrate that patients with low HGB or HCT values may exhibit unexpectedly low HUs within areas of intracranial hemorrhage. ${ }^{8}$ Our results confirm, by using a large, prospective patient series imaged by using modern CT equipment, these previously suspected correlations in the intracranial sinuses.

There are several limitations of this study. We are uncertain to what extent, if any, the diameter and attenuation of the skull may alter intracranial HU determination. Also, we did not systematically account for the subject's hydration status by examining creatinine or blood urea nitrogen levels. We believe that by using a prospective, consecutive patient population with 166 subjects that we achieved a reasonable and clinically applicable range of patients that probably minimized any such unforeseen variables that could introduce error. Because HUs vary slightly from scanner to scanner, only 2 scanners were used for this study. ${ }^{9}$ It is conceivable, though unlikely, that a subject categorized without thrombosis had an asymptomatic, undiagnosed clot. It is important to point out that a normal HU does not exclude the presence of a clot in the venous sinuses and that in the proper clinical setting of a patient with unexplained symptoms and possible risk factors for clot, further definitive imaging studies should be used despite a negative head CT.

The small number of cases with sinus thrombosis limits the precision of conclusions regarding a reliable cutoff margin for gauging concern for possible thrombosis based only on HCT and $\mathrm{HU}$ measurements. Although our findings suggest that the $\mathrm{H}: \mathrm{H}$ ratio may be helpful clinically, further, perhaps prospective, studies with higher numbers of cases will be required to demonstrate how useful the $\mathrm{H}: \mathrm{H}$ ratio may be in trying to discriminate between patients with or without sinus thrombosis.

\section{Conclusions}

Dural sinus x-ray attenuation correlates strongly with HCT and HGB. Elevated attenuation within the dural sinuses in patients without elevated HGB or HCT levels should lead to suspicion of thrombosis. None of our cases without sinus thrombosis had $\mathrm{HU}$ values of $\geq 70$, whereas 7 of 8 cases with thrombosis did. Although the low number of subjects with thromboses does not allow us to derive a reliable means of discriminating patients with thrombosis, our findings suggest that in patients with Hounsfield attenuation of $>70$, further confirmatory imaging studies may be warranted to exclude the possibility of sinus thrombosis. In addition, even in patients with a maximal $\mathrm{HU}<70$, the $\mathrm{H}: \mathrm{H}$ ratio may be helpful in determining whether further imaging studies are warranted to exclude sinus thrombosis, but further studies will be required to fully understand its clinical utility.

\section{References}

1. Stein J, Huerta K. Ask CalJEM: when looking at a non-contrast head CT, what actually appears white in an acute hemorrhagic stroke? Cal J Emerg Med 2002;3(4):70-71

2. Collins AJ, Gillespie S, Kelly BE. Can computed tomography identify patients with anaemia? Ulster Med J 2001;70:116-18

3. Ben Salem D, Osseby GV, Rezaizadeh-Bourdariat K, et al. Spontaneous hyperdense intracranial vessels seen on CT scan in polycythemia cases. J Radiol 2003;84:605-08

4. New PF, Aronow S. Attenuation measurements of whole blood and blood fractions in computed tomography. Radiology 1976;121:635-40

5. Nelson MD Jr, Thompson JR, Hinshaw DB Jr, et al. Radiodense dural sinuses: new CT sign in patients at risk for hypoxemic insult. AJNR Am J Neuroradiol 1981;2:545-48

6. Cobelli R, Zompatori M, De Luca G, et al. Clinical usefulness of computed tomography study without contrast injection in the evaluation of acute pulmonary embolism. J Comput Assist Tomogr 2005;29(1):6-12

7. Healy JF, Nichols C. Polycythemia mimicking venous sinus thrombosis. AJNR Am J Neuroradiol 2002;23:1402-03

8. Kasdon DL, Scott RM, Adelman LS, et al. Cerebellar hemorrhage with decreased absorption values on computed tomography: a case report. Neuroradiology 1977;13:265-66

9. Bydder GM, Kreel L. The temperature dependence of computed tomography attenuation values. J Comput Assist Tomogr 1979;3(4):506-10 